\title{
Über den Lebenszyklus der marinen Planktondiatomee Stephanopyxis turris (Centrales) und seine Steuerung im Experiment ${ }^{1}$
}

\author{
Gerhard Drebes \\ Botanisches Institut der Universität Marburg
}

On the life cycle of the marine plankton diatome Stephanopyxis turris (Centrales) and its experimental manipulation. Anmerkung der Schriftleitung: DREBES Beitrag zum Vierten Meeresbiologischen Symposion, Hamburg-Altona, 28.-31. Oktober 1963, wird hier nur als Kurzfassung wiedergegeben. Eine ausführliche Darstellung erfolgt zu einem späteren Zeitpunkt.

Ein Klon von Stephanopyxis turris (GREv. \& ARN.) RALfS aus der Nordsee wird seit 10 Jahren kultiviert. Zellteilung, Meiosis, Befruchtung und Auxosporenbildung mit ihren metagamen Mitosen sowie Entstehung und Keimung der Dauersporen wurden an lebendem Material mit Eintauchsystemen studiert. Die Alge ist monözisch, wie alle anderen bisher untersuchten Centrales. Die männlich determinierte Zelle bildet in 2 bis 3 Teilungsschritten 4 oder 8 in der Mutterzelle eingeschlossene, beschalte Spermatogonien, während das Oogon direkt aus einer vegetativen Zelle hervorgeht. Die Spermien entstehen merogen (Melosira-Typ). Die Reduktionsteilungen in den eineiigen Oogonien erfolgen ohne Zy tokinese. Durch Überdehnung und durch leichtes Abknicken der Oogonien wird zwischen den freien Enden der beiden Gürtel ein Fleck Plasmalemma entblößt, durch das das Spermium eindringt. In der Auxospore, der sich maximal auf fast $100 \mu$ aufblähenden Zygote, findet im Zusammenhang mit der Ausscheidung der beiden Erstlingsschalen je eine metagame Mitose statt. Die Sexualisierung der Alge kann durch plötzliche Steigerung der Lichtintensität, etwa von $250 \mathrm{Lux}$ auf 2500 Lux, hervorgerufen werden; eine gleichzeitige Temperaturerhöhung von $15^{\circ}$ auf $21^{\circ} \mathrm{C}$ wirkt fördernd aber nicht für sich auslösend. 10- bis 14stündiger "Lichtschock" genügt für reichliche Auxosporenbildung binnen 48 Stunden. Die Entstehung der Gametangien ist abhängig von der Zellenbreite und findet von $45 \mu$ abwärts, also bei relativ schmalen Zellen, statt. Bei höherer Zellenbreite ist dabei die Oogonien-, bei niederer die Spermatogonienbildung gefördert. $\mathrm{Zu}$ Dauersporen können beliebig breite Zellen bis herab zu $30 \mu$ (die kleinsten existenzfähigen Zellen messen $10 \mu$ ) bei tiefen Temperaturen $\left(9^{\circ}-18^{\circ} \mathrm{C}\right)$ werden, wobei niedere Lichtintensitäten (300-1000 Lux) fördernd wirken. Die Keimung erfolgt bevorzugt bei höheren Temperaturen $\left(21^{0}-24^{\circ} \mathrm{C}\right)$, sowohl im Licht als auch bei Dunkelheit. Frisch gebildete Dauersporen brauchen eine Ruhezeit von

${ }^{1}$ Brief outline of main results; a complete report will be published later. 
mindestens vier Tagen, ehe sie keimfähig sind. Unter anderem ungelöst ist das entwicklungsphysiologisch interessante Problem der Spontandifferenzierung, der Frage, welche Bedingungen verantworten, daß eine bestimmte Zelle einer Kolonie an einem bestimmten Tage sexualisiert und nicht - wie eventuell ihre Nachbarn-vegetativ bleibt.

\section{Diskussion im Anschluß an den Vortrag DReBEs}

v. Sтовсн: Ich möchte besonders auf die Gunst von Stephanopyxis als Experimentier- und Kursobjekt hinweisen, sowie darauf, daß die Untersuchungen sowohl hinsichtlich der Wirkung der physikalischen Faktoren als auch der Nährstoffe erweitert werden sollen. 